\title{
How to Integrate Mental Health and HIV Services: A Social-Ecological Approach
}

\section{Hanna Schweitzer, MPH*, Suzanne Leclerc-Madlala, PhD}

United States Agency for International Development, Washington, DC, USA

*Corresponding Author: Hanna Schweitzer, United States Agency for International Development, Washington, DC, USA

Received date: 25 August 2021; Accepted date: 07 September 2021; Published date: 10 September 2021

Citation: Schweitzer H, Leclerc-Madlala S (2021) How to Integrate Mental Health and HIV Services: A Social-Ecological Approach. J Comm Med and Pub Health Rep 2(8): https://doi.org/10.38207/JCMPHR/2021/0208148

Copyright: (C) 2021 Hanna Schweitzer. This is an open-access article distributed under the terms of the Creative Commons Attribution License, which permits unrestricted use, distribution, and reproduction in any medium, provided the original author and source are credited.

\section{Background}

Calls by the global health community to pay closer attention to the mental health needs of people living with HIV (PLHIV) have grown considerably. There is substantial evidence that impairment in mental health leads to negative health outcomes for PLHIV at each step in the care continuum, starting with diagnosis and extending to achieving viral suppression.[1] In one systematic review that looked at predictors of loss to follow-up (LTFU) from antiretroviral therapy (ART) among adults in sub-Saharan Africa, patients who had mental illness had higher odds of LTFU from the ART program compared to their counterparts.[2] Currently, there are very few health services in low-to-middle income countries (LMICs) that address the needs of people managing the co-occurrence of HIV and mental health. Unequal distribution of these limited resources heightens the problem of poor access to mental health care. The majority of healthcare in LMICs is based in main urban areas often not accessible to the rural populations [3]. This needs to change. It is common practice for mental health care to be delivered by nurses trained in mental health.[4] However, given the lack of existing mental health resources-especially in poor countries heavily burdened by HIVand given the urgency and scale of the unmet need, we must look for innovative ways to address the problem. In addition, many LMICs have significant strengths such as families and community support. These strengths could be harnessed to equip families with skills to become extended therapy providers and can mitigate against the lack of available trained practitioners in LMICs [3]. The time is now to integrate mental health treatment interventions into HIV prevention, testing, and treatment.

HIV imposes a major psychological burden on individuals. PLHIV are three times more likely than the general population to have mental disorders. [5] It is estimated that HIV prevalence among people living with severe mental disorders is between $1.5 \%$ in Asia and up to 19 $\%$ in Africa.[6] Studies across 38 countries show that $15 \%$ of adults and $25 \%$ of adolescents living with HIV reported depression, which could deter adherence. [6] By 2030, depression is likely to be the third leading cause of disease burden in low-income countries and the second highest cause of disease burden in middle-income countries. The proportions of people with mental illness who receive evidencebased treatments are likely to be under $11 \%$ [3]. PLHIV often suffer from common mental disorders as they adjust to the impact of an HIV infection diagnosis; the difficulties of living life with a chronic illness; a shortened life expectancy; and the need to take medications every day, stigmatization, and loss of social support. [7]PLHIV often experience mental health issues that lead them to stop seeking or continuing health care and to be less adherent to treatment. [6] The presence of depressive symptoms has been shown to be associated with a $42 \%$ lower likelihood of achieving antiretroviral (ARV) adherence. [6] This provides sufficient evidence for why depression should be addressed within the context of HIV; depressed individuals are much less likely to adhere to antiretroviral treatment regimens when compared with PLHIV not experiencing depression. [8] Integrating mental health within HIV work is not only logical but in the long run, will improve treatment and retention in care.

Mental health impairment that results in significant levels of psychiatric distress can interfere with regular HIV testing as well as successfully linking to HIV healthcare, staying in care, initiating ART, and remaining adherent to ART to achieve HIV viral suppression. [1] Innovative, effective, and low-cost solutions for addressing mental health in LMICs in the context of HIV are urgently needed. However, finding solutions that are simple and sustainable will not be straightforward, as the issues are multifaceted and intertwined. A viable starting point is to consult public health frameworks that provide a more holistic understanding of the problem, such as the Socio-Ecological Model (SEM). Health providers and project planners can gain insight into the dynamic interrelations among various personal and environmental factors that shape the bidirectional relationship of HIV and mental health. Such insights are foundational for developing well-tailored and theorygrounded mental health and HIV interventions. 


\section{The Socio-Ecological Model}

The socio-ecological model is recognized as a systematic and coordinated approach for understanding disease risks, particularly among underserved and vulnerable population groups. [9] Symptoms from mental illness are a driver for treatment nonadherence. Selfreported depression and alcohol misuse were among the top 15 barriers to adherence. ${ }^{1}$ The likelihood of achieving good (80 \%) ART adherence was $42 \%$ lower among those with symptoms of depression than those without them. This finding was consistent across low-, middle-, and high-income countries [10]. Ultimately, the goal is to improve HIV treatment engagement and a holistic approach will be key to achieving good treatment engagement. The SEM posits that there are multilevel and interactive factors such as individual, community, institutional and policy factors that impact access and acceptability of mental health in HIV care. Programs designed around SEM constructs consider the synergies between various contextual factors and link individual's health behavior with their lived experiences. Individual-level factors include personal beliefs around HIV, shame, stigma, age, gender, marital status, perceived social norms, and substance use and all may impact whether or not an individual will seek mental health services. Interpersonal level factors

\section{Recommendations}

Effective solutions for integrating mental health into HIV care would also have to include educating providers and the community around stigma and mental health. However, merely educating providers on the symptomatology of depression, anxiety, and other common mental illnesses so that they know what to look for in their clients may not be sufficient. There is a need to incorporate more culturally resonating, locally relevant knowledge and conceptualizations of mental health, as well as the narratives of key stakeholders impacted by mental disorders. In many communities, there is a stigma surrounding mental illness that is often just as strong as the stigma surrounding HIV. [12] Providing practitioners with screening tools and education manuals about mental health along with case studies of community members coping with different mental illnesses could reduce the stigma associated with mental illness within communities. This way, providers can help normalize mental illness within communities and be equipped to provide knowledge and resources to clients. In addition, incorporating social support and community

\section{Conclusion}

There are limited mental health services and interventions addressing the needs of PLHIV who experience mental health issues. In order to change this, the SEM can be used to gain foundational knowledge and identify points of mental health services intervention that address some of the challenges and barriers to providing mental health care. Peer-to-peer support models, faith-based organizations, and include social support, and trust, as well as communication skills and self-efficacy. Social support, for example, has been shown to guard against stress-related or stress-inducing crises, which can not only negatively impact the mental health of PLHIV but serve as a barrier to them seeking testing and treatment. [5] It is therefore crucial that health care providers assess levels of social support among their HIV clients. In addition to HIV testing service providers, involving the community will be key to scaling up and implementing mental health services. Community participation can be used to improve mental health services' cultural competency by increasing the mental health literacy of community health workers [11]. Transportation to clinics and support from faith leaders and traditional healers, as well as community-specific culture and attitudes, are all community-level factors that influence the creation and utilization of mental health services in the HIV treatment and prevention cascade. Healthy system/institutional level factors at play include provider education, training, financial contributions to mental health, and technology access. Using the SEM, interventions around mental health can be tailored to meet the unique needs of countries, communities, and priority populations.

support systems into the clinical cascade would be an important step to improving treatment and retention in care. Equipping providers with a list of support group resources on hand could connect people who have perceived low-social support. Peer-to-peer mentoring support programs provide an opportunity for PLHIV who have achieved significant recovery to assist others in their recovery journeys. Peer specialists model recovery, teach skills, provide a trusting safe space, and offer emotional support to help people experiencing mental health challenges lead meaningful lives in the community. Furthermore, faith-based organizations and traditional leaders and healers historically have been trusted by community members and used as vehicles for disseminating information, tools for organizing the community, and conduits for providing or promoting HIV services. Faith-based organizations can serve as a powerful resource for modeling mental health integration in HIV services while strengthening existing community-level infrastructure.

community-wide education campaigns are all effective and viable avenues for initiating this integration process. In order to successfully bridge mental health services with HIV care, we suggest looking to the SEM to place PLHIV at the center of our program design to help improve health outcomes and control the HIV epidemic. 
Declaration of interest: The contents in this article do not necessarily reflect the view of the U.S. President's Emergency Plan

\section{References}

1. Remien RH, Stirratt MJ, Nguyen N, Robbins RN, Pala AN, et al. (2019) Mental health and HIV/AIDS: the need for an integrated response. AIDS. 33(9): 1411-1420.

2. Kebede HK, Mwanri L, Ward P, Gesesew HA (2021) Predictors of loss to follow up from antiretroviral therapy among adults in sub-Saharan Africa: a systematic review and meta-analysis. Infect Dis Poverty. 10(1): 33.

3. Rathod S, Pinninti N, Irfan M, Gorczynski P, Rathod P, et al. (2017) Mental Health Service Provision in Low- and MiddleIncome Countries. Health Services Insights. 10: 1178632917694350

4. Wogrin C, Willis N, Mutsinze A, Chinoda S, Verhey R, et al. It helps to talk: A guiding framework (TRUST) for peer support in delivering mental health care for adolescents living with HIV. PLOS ONE/ 2021; 16(3): e0248018.

5. Ezeanolue EE., Iheanacho T, Adedeji, IA, Itanyi IU, Olakunde B, et al. (2020) Opportunities and challenges to integrating mental health into HIV programs in a low- and middle-income country: insights from the Nigeria implementation science Alliance. BMC Health Serv Res 20: 904.

6. Unaids.org. Better integration of mental health and HIV services is needed. UNAIDS. October 10, 2018. for AIDS Relief, or the U.S. Agency for International Development or the U.S. Government.

7. 7 Duko, B, Toma, A, Abraham, Y (2019) Prevalence and correlates of common mental disorders among HIV patients attending antiretroviral therapy clinics in Hawassa City, Ethiopia. Ann Gen Psychiatry. 18: 17.

8. Meffert SM, Neylan TC, McCulloch CE, Maganga L, Adamu Y, et al. (2019) East African HIV care: depression and HIV outcomes. Glob Mental Health (Camb). 6: e9.

9. Mahadevan M, Amutah N, Ramos LJ, Raines ER, King J, et al. (2014) Project THANKS: A Socio-Ecological Framework for An Intervention Involving HIV Positive African American Women with Comorbidities. Journal of Health Disparities Research and Practice. 7(7): 2.

10. Uthman OA, Magidson JF, Safren SA, Nachega JB (2014) Depression and adherence to antiretroviral therapy in the low-, middle- and high-income countries: a systematic review and meta-analysis. Curr HIV/AIDS Rep. 11(3): 291-307.

11. Rajabzadeh V, Burn E, Sajun SZ, Suzuki M, Bird VJ, et al. (2021) Understanding global mental health: a conceptual review. BMJ Global Health. 6: e004631.

12. Monteiro, NM (2015) Addressing Mental Illness in Africa: Global Health Challenges and Local Opportunities. Community Psychology in Global Perspective CPGP, Comm. Psych Glob. Persp. 1(2): 78-95. 